\title{
Publisher Correction: Spatiotemporal control of laser intensity
}

Dustin H. Froula (1), David Turnbull, Andrew S. Davies, Terrance J. Kessler, Dan Haberberger, John P. Palastro, Seung-Whan Bahk, Ildar A. Begishev, Robert Boni, Sara Bucht, Joseph Katz and Jessica L. Shaw

Correction to: Nature Photonics https://doi.org/10.1038/s41566-018-0121-8, published online 12 March 2018.

In the version of this Letter originally published, in the first sentence of the Methods, ref. ${ }^{31}$ was incorrectly cited; it should have been ref. ${ }^{32}$. This has now been corrected.

\section{Publisher Correction: Universal impedance matching and the perfect transmission of white light}

Ku Im (D), Ji-Hun Kang (iD and Q-Han Park

Correction to: Nature Photonics https://doi.org/10.1038/s41566-018-0098-3, published online 26 February 2018.

In the version of this Letter originally published, in equation (2), the vectors ' $\vec{E}$ ' and ' $\vec{H}$ ' should have appeared as 'E' and ' $\mathbf{H}$ '; equations (3)-(7) and (11) appeared incorrectly, they were broken at the wrong places; in Fig. 1b, in the right panel, the equation was wrong and should have read ' $\varepsilon_{z} / \varepsilon_{0}=\alpha \mu_{z} / \mu_{0}$ '; and in Fig. $2 \mathrm{~b}$, there was an artefact in the $t=4$ image labelled 'Total, Transmission $=0.994$ ' as well as a white line separating all $t=3$ images from the $t=4$ images. These errors have now been corrected in the online versions.

\section{Publisher Correction: Massively parallel sensing of trace molecules and their isotopologues with broadband subharmonic mid-infrared frequency combs}

\section{A. V. Muraviev, V. O. Smolski, Z. E. Loparo and K. L. Vodopyanov}

Correction to: Nature Photonics https://doi.org/10.1038/s41566-018-0135-2, published online 26 March 2018.

In the version of this Article originally published, in equation (9), the ' $\Delta$ ' in the first ' $\Delta f_{\text {rep }}$ ' shouldn't have been included; the equation should have read

$$
\nu_{m}=\left(\nu_{1 m+1}+\nu_{2 m}\right) / 2=\Delta_{1} / 2+\left(m+\frac{1}{2}\right) f_{\text {rep }}-\frac{\Delta f_{\text {rep }}}{4}
$$

And, in equation (10), within the brackets, $f_{\text {rep }}$ and $f$ were the wrong way round; the equation should have read

$$
\nu=\Delta_{1} / 2+\frac{f_{\text {rep }}}{\Delta f_{\text {rep }}}\left(f_{\text {rep }}-f\right)-\frac{\Delta f_{\text {rep }}}{4}
$$

These equations have now been corrected in the online versions. 